\title{
Testing Microgravity Flight Hardware Concepts on the NASA KC-135
}

Susan M. Motil, Angela R. Harrivel, and Gregory A. Zimmerli Glenn Research Center, Cleveland, Ohio

Prepared for the

39th Aerospace Sciences Meeting and Exhibit sponsored by the American Institute of Aeronautics and Astronautics Reno, Nevada, January 8-11, 2001

National Aeronautics and Space Administration

Glenn Research Center 


\section{Acknowledgments}

We gratefully acknowledge the assistance of E. Hovenac, D. Minjares, and R. Skupinski for their effort in designing and developing the KC-135 aircraft test rig. This work was supported by NASA through the Microgravity Science Division.

This report is a formal draft or working paper, intended to solicit comments and ideas from a technical peer group.

This report contains preliminary
findings, subject to revision as
analysis proceeds.

Available from

NASA Center for Aerospace Information 7121 Standard Drive

Hanover, MD 21076

Price Code: A03
National Technical Information Service 5285 Port Royal Road Springfield, VA 22100

Price Code: A03 
TESTING MICROGRAVITY FLIGHT HARDWARE CONCEPTS ON THE NASA KC-135

\author{
Susan M. Motil, Angela R. Harrivel, and Gregory A. Zimmerli \\ National Aeronautics and Space Administration \\ Glenn Research Center \\ Cleveland, Ohio 44135
}

\begin{abstract}
$\underline{\text { Abstract }}$
This paper provides an overview of utilizing the NASA KC-135 Reduced Gravity Aircraft for the Foam Optics and Mechanics (FOAM) microgravity flight project. The FOAM science requirements are summarized, and the KC-135 test-rig used to test hardware concepts designed to meet the requirements are described. Preliminary results regarding foam dispensing, foam/surface slip tests, and dynamic light scattering data are discussed in support of the flight hardware development for the FOAM experiment.
\end{abstract}

\section{$\underline{\text { Introduction }}$}

The Principal Investigator of the FOAM flight experiment is Professor Douglas Durian from the University of California, Los Angeles. The experiment is planned to be conducted on board the International Space Station in 2006. The objective of the FOAM experiment is to relate microscopic information about foam structure and dynamics to the macroscopic properties such as foam rheology and stability.

Because primary interest is in wet foams, long duration microgravity conditions are needed so that the primary science goals can be achieved. Fulfilling the FOAM science objectives requires a general understanding of the effects of a low gravity environment on the behavior of foam and hardware functionality. Utilizing reduced gravity facilities, such as the NASA KC-135, can enhance the development process of space flight hardware. ${ }^{1}$ Hardware that performs flawlessly in a $1 \mathrm{~g}$ environment will often fail in a low gravity environment ( $g=9.8 \mathrm{~m} / \mathrm{s}^{2}$ is the acceleration due to Earth's gravity).

A series of low gravity tests were conducted on board the NASA KC-135 Reduced Gravity Aircraft in support of the FOAM microgravity flight project. The FOAM test rig was developed to take advantage of the flexibility and availability of the KC-135, providing the team with otherwise unobtainable data. Design of the test rig (Figure 1) was modularized for versatility. Hardware components could be exchanged to meet specific test objectives.

The KC-135 tests enabled the FOAM flight hardware development team to obtain quantitative results on sample cell surface treatments, laser light scattering, and video microscopy measurements. In addition, qualitative tests were conducted to observe the production of wet foams, and evaluate various foam delivery systems.

In the first series of $\mathrm{KC}-135$ flights, the FOAM project team conducted tests to determine compatible sample-cell surface treatments for future rheology tests, and to dispense foam in a controlled, repeatable manner in a low gravity environment. Test results were obtained on selected surface treatments and materials to determine the combination that allowed the least amount of slippage between the foam and the plate surface. Results of these tests will assist the project team in their selection of a material and surface treatment for the design of a rheometer sample cell fixture that will eventually contain the sample for the FOAM flight experiment. Initial observations on foam dispensing were evaluated, and lead the project team to further testing options.

The second series of KC-135 testing was conducted to further test a "bag-in-can" foam dispensing concept, and to conduct laser light scattering measurements on wet foams. The FOAM test rig was reconfigured to accommodate a video microscopy system to observe the bubbles while conducting simultaneous laser light scattering measurements. Valuable data was successfully taken with the light scattering system during low $\mathrm{g}$ $(\sim 0.01 \mathrm{~g})$, Lunar-g (1/6 g), Martian-g $(\sim 0.4 \mathrm{~g})$ and highgravity $(1.5-2 \mathrm{~g})$ portions of the $\mathrm{KC}-135$ flights. 
The $\mathrm{KC}-135$ tests have provided the FOAM project team with essential information regarding foam behavior. Flight hardware development for the FOAM experiment presents numerous challenges to the engineering team. Despite the short duration ( $20 \mathrm{~s})$ of low gravity provided by the $\mathrm{KC}-135$, much can be learned regarding foam behavior, and assessment of hardware concepts. ${ }^{2}$ Results from the KC-135 Reduced Gravity Aircraft will support the development process, and help verify the functionality of the hardware in meeting the FOAM science requirements.

\section{Foam Science Requirements}

The FOAM microgravity experiment seeks to provide a fundamental understanding of foam rheology and stability in relationship to the foam bubble structure and dynamics. This is to be accomplished through the use of light scattering measurements, rheological measurements, and video microscopy on foams of variable liquid content. The developed instrumentation must meet the science requirements for all four of these elements. Throughout the experiment, the foam will be allowed to coarsen ${ }^{3}$ and data will be collected in each of the required diagnostic techniques.

Of particular interest is the loss of rigidity in foams with a high liquid content, i.e., wet foams. The study of wet aqueous foams is particularly difficult in normal gravity because the liquid easily drains out of the sample. ${ }^{4.5}$ In this particular experiment, the liquid content is required to range from $5 \%$ to $40 \%$. The sample cell containing the foam must accommodate simultaneous video microscopy, diffusing-light spectroscopy, and rheological measurements. The sample cells are required to be loaded with foams without introducing large gas pockets and other inhomogeneities. In addition, the sample cell must be designed such that uniform shear deformation throughout the foam sample can be achieved.

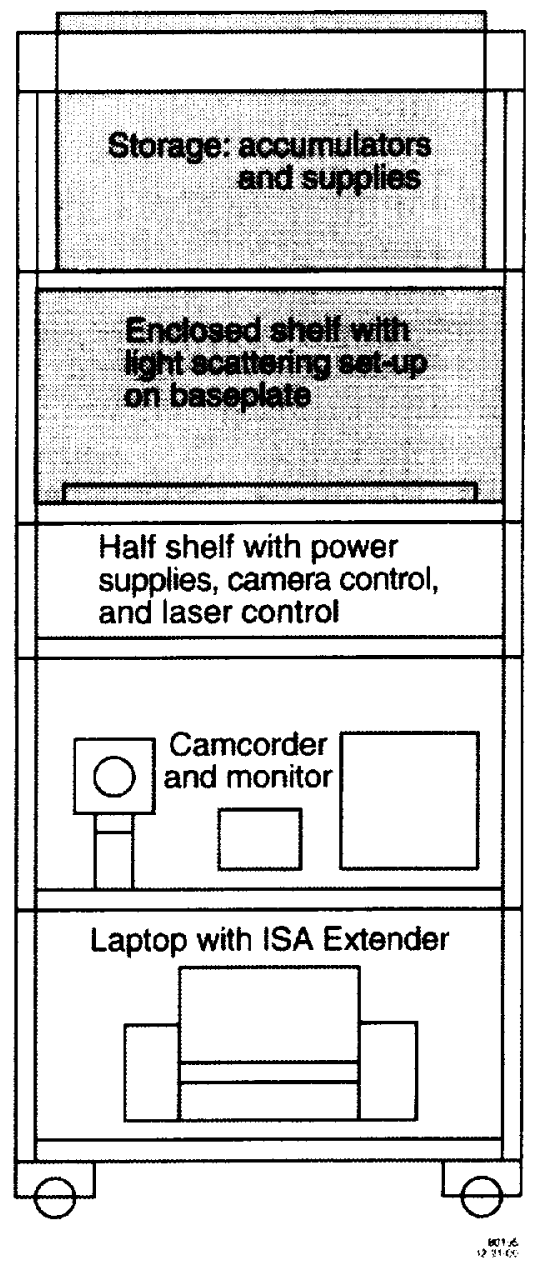

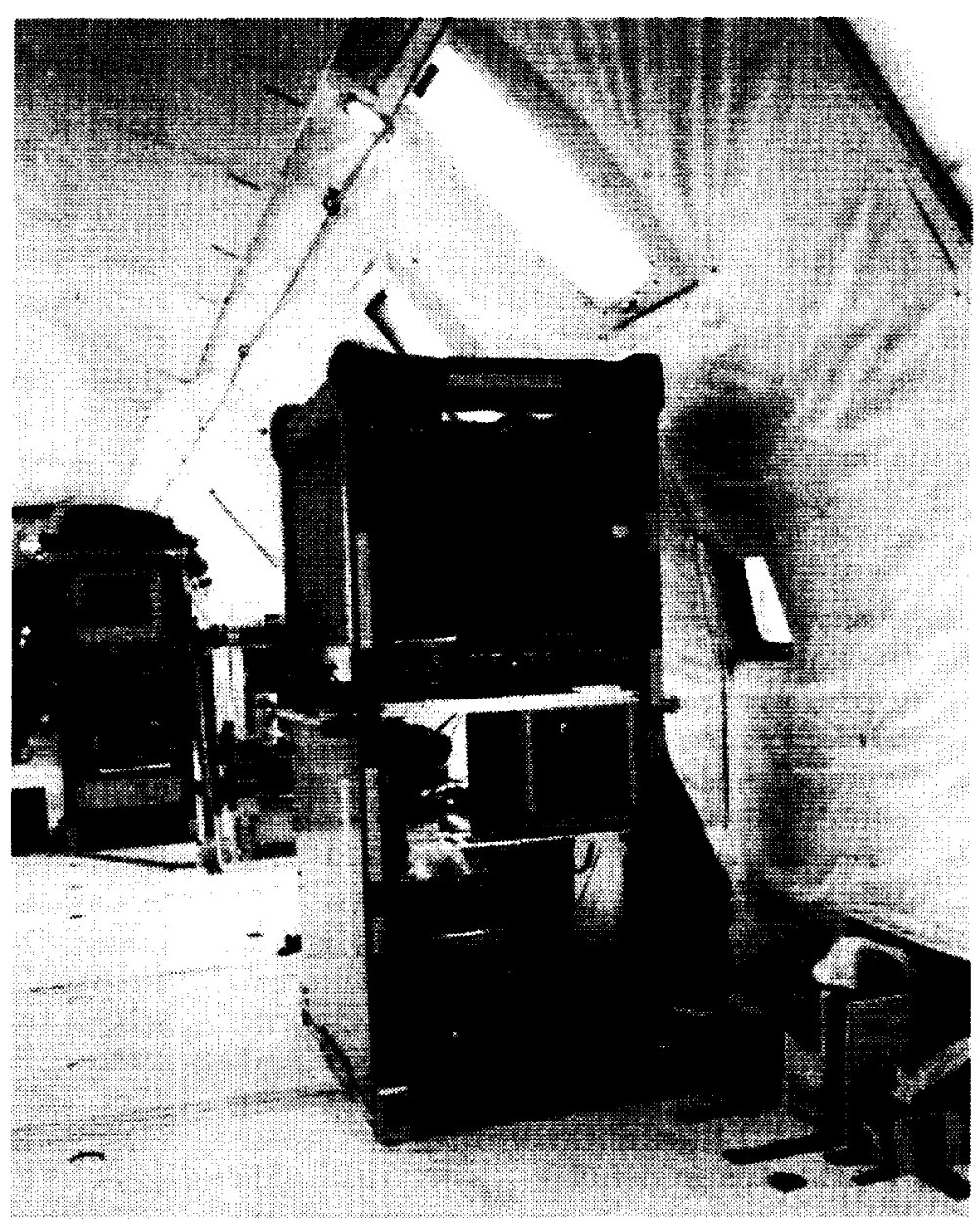

Figure 1.-FOAM KC-135 aircraft rig. 
Rheological measurements are made throughout the foam coarsening process. A cone-and-plate sample cell geometry is desired because it will provide a uniform shear rate, and allows for easy cleaning of the sample. In addition, this geometry provides access to diffusinglight spectroscopy measurements at different foam thicknesses.

Video microscopy will be used to record the gas bubbles at the surface. Bubble size and distribution can be determined through this method. Recordings are required throughout the aging process (coarsening) of the foam sample.

These techniques have been demonstrated with dry foams in ground-based studies. ${ }^{6.7 .8 .9}$ Microgravity conditions are necessary to eliminate drainage for wet foams such that observations of the changes in structure, rearrangement dynamics, coarsening, and rheology that are expected as liquid content increases can be achieved.

\section{$\underline{\text { KC-135 Hardware and Test Results }}$}

Throughout the flight hardware development process, it is important to show that concepts meet the FOAM science requirements. The FOAM test rig was designed to be compatible with the NASA KC-135 Reduced Gravity Aircraft. ${ }^{10}$ It is a standard aircraft instrument rack that was reinforced and outfitted with interfaces for mounting in the KC-135. Handles and casters were installed to facilitate moving the rack from place to place.

Most instruments used in the experiment were commercial off-the-shelf hardware and attached to the base plates. The base plates mount to the racks via adjustable shelves, which aid in the versatility of the test rig. All electronic components use 120 VAC power, which was compatible with the power supply from the $\mathrm{KC}-135$ aircraft.

The experiment was designed to be operated by one person. Tests conducted using this rig were foam dispensing, slip tests, video microscopy, and laser light scattering. Video data from all tests were recorded on digital $8 \mathrm{~mm}$ tape. A color monitor was used to verify that the system was operating correctly.

During the $\mathrm{KC}-135$ flights the parabola numbers were noted, as each parabola provides near $0 \mathrm{~g}$, but each differs due to pilot technique and weather conditions. Data is provided, via an accelerometer system on board the aircraft, which measures the g-forces along three axes. This data can be correlated with the project flight data to differentiate Martian and Lunar $g$ simulations from $0 \mathrm{~g}$ parabolas, or to identify atypical noisy parabolas.

\section{Foam Dispensing Hardware}

The FOAM flight experiment requires testing foams with various liquid contents. As the volume fraction of liquid is increased, the foam becomes wetter and behaves more like a simple liquid. In order to produce and conduct experiments on foams with various liquid fractions, a method of consistently dispensing foams at specific liquid contents in microgravity will be required.

The simple task of dispensing and containing a stream of foam is challenging in the microgravity environment. In order to observe the various dispensing methods, a video camera mounted on the $\mathrm{KC}-135$ rig was used to capture the results during the first series of tests. Videotape provided a convenient method of recording the qualitative, visual results. In addition, a microphone was employed to capture audio notes to eliminate the need for written observations during the flight.

Low gravity tests were conducted to simply observe what happens as foam is dispensed from a can of shaving cream. Foam was generated using commercially provided aerosol cans, and a reusable bag-in-can system. Inside a can of shaving cream are a mixture of water, surfactants, propellant, and other additives. A typical propellant is A-31, which contains a propaneisobutane mixture that condenses at 31 psi. A dip-tube extends from the exit nozzle to the bottom of the can, where the liquid mixture is extracted. The placement of the tube relies on gravity to force liquid to the bottom of the can, and suggests that it will be ineffective in low gravity. When the foam is dispensed, the propellant vaporizes and forms bubbles of propane-isobutane gas in the water/surfactant mixture. A typical can of commercial shaving cream produces a foam having a liquid fraction of 7 to $10 \%$. The liquid content of the foam was adjusted by varying the water/propellant ratio in the initial mixture.

The reusable bag-in-can system was employed during the second series of tests. These were tethered directly to the rig for safety during testing and otherwise stored in the top shelf bin. This system consisted of a bladder (bag) filled with the foam mixture. In this design, a foil bag is filled with a water and surfactant mixture. The bag is presealed, but contains a nozzle and valve. The bag is placed in a can, which is sealed and then pressurized to a pressure below the vapor pressure of the propellant. The propellant is then forced through the nozzle into the bag 
by its own pressure, and finally the can is pressurized to a pressure well above the saturated vapor pressure of the propellant. By creating an overpressure environment on the outside of the bag, the propellant remains in a condensed state even as the sample is depleted. Since the gas is separated from the liquid phase, only the liquid can be expelled from the can.

\section{Foam Dispensing Test Results}

Our simple tests were to continuously dispense foam from an aerosol can during the low $\mathrm{g}$ parabolas, and record the observations by video. We found that foam dispenses intermittently with pockets of gas, producing an unacceptable stream at the output. The gas pockets are ejected when the vapor pocket in the can reaches the dip-tube. In low gravity, the liquid in the can was observed to easily move away from the dip-tube, causing chaotic streaming of the foam out of the nozzle.

To circumvent the problem of a vapor pocket inside the can containing the foam sample, an alternative method of dispensing the foam using the "bag-in-can" method was tested. Tests in $1 \mathrm{~g}$ confirmed that the bag-in-a-can method produced a foam stream irrespective of the orientation of the can. We did find, however, that in order for the technique to work, sufficient overpressure in the can was needed to keep the foil bag collapsed. A drawback of the added pressure is that the foam dispenses much faster. Nevertheless, we confirmed that this design also worked in a low gravity environment.

\section{Surface-Slip Hardware}

The rheology measurements planned for the flight experiment require that the surfaces of the rheometer in contact with the foam sample provide a no-slip condition, so that the sample is sheared in a uniform and consistent manner. The purpose of this KC-135 experiment was to test various surface treatments with wet foams, and compare the results with ground-based observations. The plate materials tested included smooth and sandblasted glass, smooth and sandblasted acrylic, acrylic randomly scored with a knife edge, sandblasted quartz, and sandblasted polycarbonate. The bottom plate was held fixed while the top plate was translated horizontally by a motorized linear stage at $375 \mu \mathrm{m} / \mathrm{s}$. The top plate holder was designed for easy change out of the top plate material. Slip tests were performed using a $1 \mathrm{~mm}$ gap. A sponge was located below the two plates that caught fluid pulled from between them during the high-g pull out portions of the flights. This hardware configuration allowed for observations of foam slippage on the plate surface while creating a stress on the foam via movement of the top plate.

A CCD camera was rigidly attached to the moving stage and focused on the inside surface of the moving plate. This camera has a very small sensor head, with its remaining electronics and power supply located elsewhere. This feature allows the camera and lens to be located where space and weight may be limited, as is often the case with rigs designed to be flown on the KC-135. The camera was mounted vertically. A ring light was used to illuminate the sample. The camera output was routed to both the monitor and a camcorder for digital recording to tape.

An extension tube was used on the nozzle of each foam dispenser to inject the foam sample between the plates at the beginning of the $0 \mathrm{~g}$ period. The stage was set in motion, and the image of the bubbles at the inside surface of the top plate were observed and recorded. The plates were removed and cleaned between samples. Voice notations were recorded with the images, which identified the plate material, translation velocity, and the liquid content of the foam used.

\section{Surface-Slip Test Results}

We measured the apparent slip at a surface as a function of shear rate and foam liquid content using various surface treatments, in both $1 \mathrm{~g}$ and low $\mathrm{g}$. Ideally, a layer of gas bubbles would remain stuck to the walls of the container, providing a no-slip condition. Thus, surfaces that promote non-wetting of the liquid are preferred.

The smooth glass and sandblasted polycarbonate performed the worst, as they consistently showed slip of the bubbles at the surface. The sandblasted quartz and randomly scored acrylic performed the best, and often showed that the bubbles remained fixed at the surface during the 15 second test. The other materials exhibited intermediate performance.

The slip condition was tested with foams having a liquid content that ranged from $10 \%$ up to $40 \%$. There did not appear to be any correlation between slip speed and liquid content. Nor did we observe any difference in the slip behavior in $1 \mathrm{~g}$ and low $\mathrm{g}$. One problem we did observe was that the slip behavior was not always repeatable. Occasionally, the bubbles would not slip even on the intermediate performing materials. Sometimes, the bubbles would not slip until the foam had been sheared for several seconds. 
More tests will be needed to fully quantify the slip performance under higher shear rates and for longer times, but clearly the sandblasted quartz and randomly scored acrylic performed the best, and the liquid content and gravity had little, if any, effect on the performance.

\section{Video Microscopy and Diffusing Wave Spectroscopy Hardware}

In addition to the rheology measurements planned for the flight experiment, the FOAM experiment will use Diffusing Wave Spectroscopy (DWS) to characterize the microscopic bubble motion. DWS is a dynamic light scattering technique that is very sensitive to microscopic motion of the foam bubbles. Even in a static aqueous foam sample (not subjected to shear), the bubbles at any given location can be observed to undergo rearrangement events. These rearrangements occur to relieve stresses in the foam that build up as the foam coarsens. The DWS technique is sensitive to the frequency and duration of these events. The faster the decay of the intensity autocorrelation signal, the more active are the bubbles in the foam sample.

Reconfiguration of the experiment for the second series of KC-135 tests was easy due to the modularity of the rig design. The FOAM aircraft test rig was equipped to accommodate Diffusing Wave Spectroscopy (DWS) and video microscopy within an enclosure (Figure 2), similar to experiment configurations developed by the Principal Investigator. ${ }^{11}$ The video and CCD cameras were relocated, and the enclosed shelf was added.

One of the shelves was enclosed with an interlocked door on the front since a $100 \mathrm{~mW}$ frequency doubled $\mathrm{Nd}$ :YAG laser was used in the DWS tests. The interior of the enclosed shelf was lined with blackout material to absorb ambient light and keep the expanded laser beam from escaping into the plane cabin. An optical breadboard was cut to match and bolted to the shelf, and optical components were installed directly to it. These included the laser head, and an electronically controlled shutter. The shutter allowed the laser to remain on during video microscopy, which was done with a white light. The laser beam was directed into a microscope objective used to expand the beam. A mirror was used to give the beam more distance to expand, as space was quite limited on this shelf. A negative lens was used between the mirror and the foam sample to further expand the beam. A single mode fiber optic probe with a collimating lens, coupled to an avalanche photo diode, collected diffuse transmitted light for DWS analysis. A CCD camera and white light were used to record video images.

One foam sample was flown per flight. The sample cell was removed after each flight and refilled just before the next flight. It was delivered to the plane just before take-off, bolted in place and immediately tumbled until data was taken during reduced gravity.

The samples were prepared by adding water to shaving cream, and mixing the sample thoroughly. The foam sample was contained in a disc-shaped cell, $70 \mathrm{~mm}$ in diameter by $3.5 \mathrm{~mm}$ thick $(13.2 \mathrm{cc})$, with randomly scored acrylic windows and an anodized aluminum spacer ring. The windows were sealed against the spacer using o-rings. To prevent drainage of liquid in wet foams, the sample was continuously tumbled about its central axis except for those periods during which DWS data was collected.

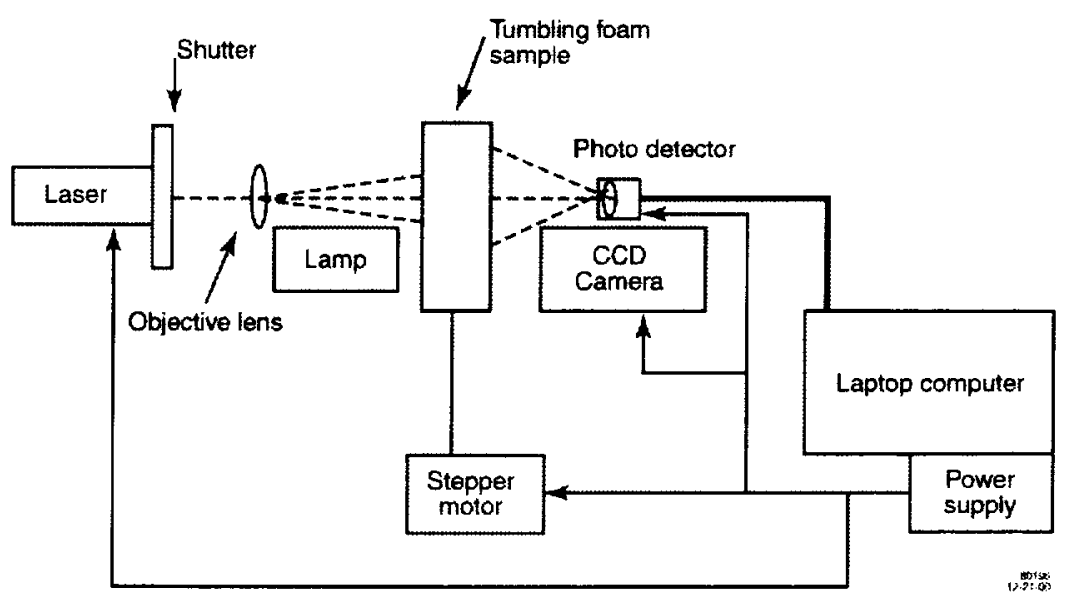

Figure 2.-Block schematic for video microscopy and laser light scattering. 
The tumbler is simply a stepper-motor-driven device and rotates the sample at a rate of once every 3 to 5 seconds. Ground-based tests showed that this technique keeps the excess liquid dispersed for several hours. At $38 \%$ liquid content, liquid begins to collect at the bottom after only two hours, even with sustained tumbling. The samples were aged in-situ for 1 to 1.5 hours while continuously tumbling.

At regular intervals, the laser shutter was closed and the avalanche photo diode turned off so the white light source could be turned on. Video images of the bubbles were recorded during these parabolas both for qualitative observation and to measure the bubble size. The laser power and tumbler speed could be easily set without opening the enclosed shelf. A set of switches on a front panel were used to control the laser, shutter, avalanche photo diode, white light, camera and tumbler. They were located across the front of the rig, in easy reach of the operator seated in front as they worked through the test procedures.

\section{Diffusing Wave Spectroscopy Test Results}

We measured the intensity autocorrelation function in several foam samples of various liquid content, as a function of g-levels. The DWS data was acquired with a correlator card installed in the docking station of a laptop PC. At the start of the $0 \mathrm{~g}$ portion of a parabola, the tumbler was turned off. Approximately 5 seconds later, a correlation function measurement was initiated. Each correlation measurement lasted 12 seconds (the low g parabola lasts about $20 \mathrm{~s}$ ). Temperature (around $25 \mathrm{C}$ ) was not controlled, and the environment is acoustically noisy.

DWS data was collected from several different g-levels: $0 \mathrm{~g}$ (closer to tens of milli-g, during the top of the parabolic loops); $1 \mathrm{~g}$ (during level flight of the aircraft): $2 \mathrm{~g}$ (during the bottom of the parabolic loops; the " $2 \mathrm{~g}$ pullout" is actually somewhere between $1 \mathrm{~g}$ and $2 \mathrm{~g}$, and varies); and also Lunar g's (1/6 g) and Mars g's (0.4g). There is a clear dependence of the characteristic decay time of $g(t)$ on the g-level (Figure 3). The data also shows that as the liquid content of the foam increases, the effect of gravity becomes more pronounced. We interpret the faster decay times to the drainage of liquid in the foam, which induces bubble motion. These results will be useful for estimating the effect of acceleration disturbances (such as thruster firings) during the microgravity flight experiment, and will also be used to bridge the gap between ground-based data and microgravity data.

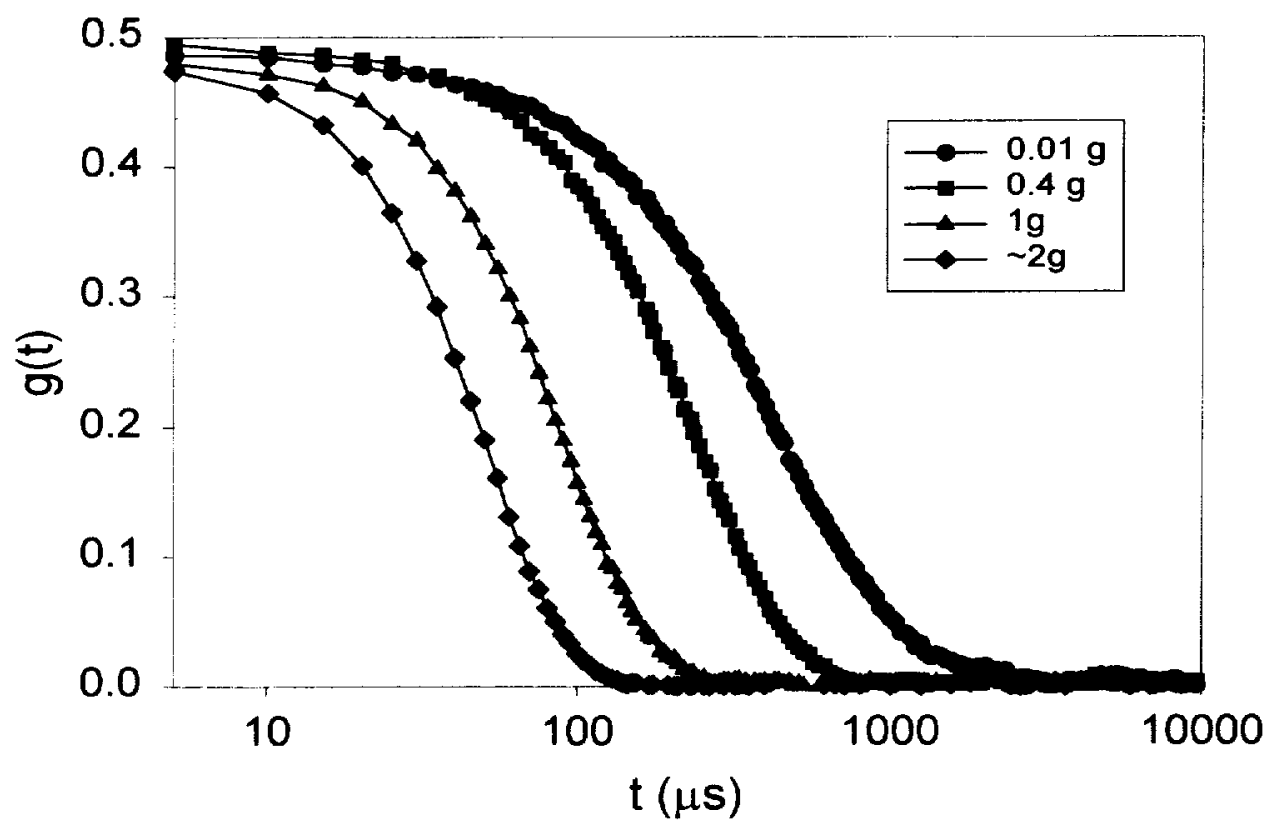

Figure 3:-DWS measurements of the intensity autocorrelation function $\mathrm{g}(\mathrm{t})$ as a function of delay time, obtained on the NASA KC-135. The foam sample contained 34\% liquid content. The legend indicates approximate g-levels. As the g-level increases, the characteristic decay time becomes shorter due to drainage and bubble motion. 


\section{Concluding Remarks}

The flight hardware development process requires an understanding of the experiment and what to expect in a microgravity environment. Engineering challenges can be overcome through a test program in both $1 \mathrm{~g}$ and low g. Although not all aspects of experimental design can be foreseen utilizing low gravity testing environments due to time limitations, many problems can be corrected prior to space flight through the use of low gravity test facilities. By conducting tests on the $\mathrm{KC}-135$ reduced gravity aircraft, we are able to foresee or debug problems that may also occur during a microgravity mission. Testing hardware on the $\mathrm{KC}-135$ low gravity aircraft offers a relatively low-cost method of testing concepts before the flight hardware design is finalized.

The $\mathrm{KC}-135$ is a versatile and unique tool, which allows researchers to perform experiments in a low gravity environment. The duration of microgravity makes it possible to complement the ground-based research, and help prepare us for our future on the International Space Station.

\section{Acknowledgements}

We gratefully acknowledge the assistance of E. Hovenac, D. Minjares, and R. Skupinski for their effort in designing and developing the $\mathrm{KC}-135$ aircraft test rig. This work was supported by NASA through the Microgravity Science Division.

\section{References}

1. V. Pletser. "Microgravity Research During Aircraft Parabolic Flights: The 20 ESA Campaigns," ESA Bulletin Nr. 82. May 1995.

2. C.A. Winter and J.C. Jones, "The Microgravity Research Experiments (MICREX) Data Base," NASA Technical Memorandum 108523 Volume I. November 1996.

3. D.J. Durian, D.A. Weitz. and D.J. Pine, "Scaling Behavior in Shaving Cream." Plys. Rev. A 36, 306-316 (1987).

4. R. Bruinsma, "Theory of Hydrodynamic Convection in Soap Films," Physica A 216, 59-76 (1995).

5. G. Verbist, D. Weaire, and A.M. Kraynik, "The Foam Drainage Equation," Joumal of Physics: Condensed Matter 8, 3715-3731 (1996).

6. D.A. Weitz, J.X. Zhu, D.J. Durian, H. Gang, and D.J. Pine, "Diffusing-Wave Spectroscopy: The Technique and Some Applications." Physics Scripta 49B, 610-626 (1993).

7. D.J. Durian, "Relaxation in Aqueous Foams," Bull. Mat. Res. Soc. 19, 20-23 (1994).

8. D.J. Durian and D.A. Weitz, "Foams," Kirk-Othmer Encyclopedia of Chemical Technology, 4 ed., edited by J.I. Kroschwitz (Wiley, New York, 1994), Vol. 11, pp. 783-805.

9. M. Tabor, J.J. Chae, G.D. Burnett, and D.J. Durian, "The Structure and Dynamics of Foams," Nonlinear Science Today (1991).

10. L.G. White, “Operations User's Guide: JSC Reduced Gravity Program User's Guide," JSC-22803 Rev. D, NASA Lyndon B. Johnson Space Center, October 2000.

11. D.J. Durian, D.A. Weitz, and D.J. Pine, "Multiple Light Scattering Probes of Foam Structure and Dynamics," Science 252. 686-688 (1991). 
Public reporting burden for this collection of information is estimated to average 1 hour per response, including the time for reviewing instructions, searching existing data sources. gathering and maintaining the data needed, and completing and reviewing the coliection of information. Send comments regarding this burden estimate or any other aspect of this collection of information, including suggestions for reducing this burden, to Washington Headquarters Services. Directorate for information Operations and Reports, 1215 Jefferson Davis Highway, Suite 1204, Arlington, VA 22202-4302, and to the Otfice of Management and Budget, Paperwork Reduction Project (0704-0188), Washington, DC 20503.

\begin{tabular}{|l|l|l}
\hline 1. AGENCYUSE ONLY (Leave blank) & $\begin{array}{c}\text { 2. REPORT DATE } \\
\text { March 2001 }\end{array}$ & $\begin{array}{c}\text { 3. REPORT TYPE AND DATES COVERED } \\
\text { Technical Memorandum }\end{array}$
\end{tabular}

4. TITLE AND SUBTITLE 5. FUNDING NUMBERS

Testing Microgravity Flight Hardware Concepts on the NASA KC-135

6. AUTHOR(S)

WU-101-44-00-00

Susan M. Motil, Angela R. Harrivel, and Gregory A. Zimmerli

7. PERFORMING ORGANIZATION NAME(S) AND ADDRESS(ES)

National Aeronautics and Space Administration

John H. Glenn Research Center at Lewis Field

Cleveland, Ohio 44135-3191

8. PERFORMING ORGANIZATION REPORT NUMBER

E-12635

9. SPONSORINGMONITORING AGENCY NAME(S) AND ADDRESS(ES)

10. SPONSORINGMONITORING

National Aeronautics and Space Administration

Washington, DC 20546-0001 AGENCY REPORT NUMBER

NASA TM-2001-210704

AIAA-2001 -0770

\section{SUPPLEMENTARY NOTES}

Prepared for the 39th Aerospace Sciences Meeting and Exhibit sponsored by the American Institute of Aeronautics and Astronautics, Reno, Nevada, January 8-11, 2001. Responsible person, Susan M. Motil, organization code 6728, $216-433-8589$.

12a. DISTRIBUTIONAVAILABILITY STATEMENT

12b. DISTRIBUTION CODE

Unclassified - Unlimited

Subject Categories: 74 and 34

Distribution: Nonstandard

Available electronically at http://gltrs.grc,nasa.gov/GLTRS

This publication is available from the NASA Center for AeroSpace Information, 301-621-0390.

13. ABSTRACT (Maximum 200 words)

This paper provides an overview of utilizing the NASA KC-135 Reduced Gravity Aircraft for the Foam Optics and Mechanics (FOAM) microgravity flight project. The FOAM science requirements are summarized, and the KC-135 testrig used to test hardware concepts designed to meet the requirements are described. Preliminary results regarding foam dispensing, foam/surface slip tests, and dynamic light scattering data are discussed in support of the flight hardware development for the FOAM experiment.

14. SUBJECT TERMS

Light scattering; Fluid flow tests in microgravity

15. NUMBER OF PAGES

SECURITY CLASSIFICATION OF REPORT

Unclassified
18. SECURITY CLASSIFICATION OF THIS PAGE Unclassified
19. SECURITY CLASSIFICATION OF ABSTRACT

Unclassified
$\mathrm{A03}$

20. UMITATION OF ABSTRACT

